\title{
UNIQUENESS OF LIMIT CYCLES IN A PREDATOR-PREY SYSTEM WITH HOLLING-TYPE FUNCTIONAL RESPONSE
}

BY

\author{
JITSURO SUGIE
}

Department of Mathematics and Computer Science, Shimane University, Matsue, Japan

\begin{abstract}
This paper is concerned with the problem of uniqueness of limit cycles in a predator-prey system with Holling's functional response $x^{p} /\left(a+x^{p}\right)$, where $a$ and $p$ are positive parameters. The problem has not yet been settled only in the case $1<p<2$. This paper gives a sufficient condition under which the predator-prey system with $1<$ $p<2$ has exactly one limit cycle by using a result of Zhang and Gao. Finally, the fact that our condition is also necessary is mentioned.
\end{abstract}

1. Introduction. We consider a predator-prey system of the form

$$
\begin{aligned}
& \dot{x}=r x\left(1-\frac{x}{k}\right)-\frac{x^{p} y}{a+x^{p}}, \\
& \dot{y}=y\left(\frac{\mu x^{p}}{a+x^{p}}-D\right),
\end{aligned}
$$

where ${ }^{\cdot}=d / d t$. Here $x$ and $y$ are the population densities of the prey and the predator, respectively, $r$ is the intrinsic growth rate of the prey, $k$ is the carrying capacity of the environment for the prey, $\sqrt[p]{a}$ is the half-saturation constant for the predator, $\mu$ and $D$ are the birth rate and the death rate of the predator. All the parameters are always positive. This system is said to have the functional response $x^{p} /\left(a+x^{p}\right)$ of Holling type and has been widely studied in many papers (for example, see $[1,2,3,4,5,6,8,9,10]$ ). Let

$$
\lambda_{p}=\sqrt[p]{\frac{a D}{\mu-D}} \quad \text { and } \quad \nu_{p}=\frac{r \mu}{D}\left(1-\frac{\lambda_{p}}{k}\right) \lambda_{p}
$$

In case

$$
\mu>D
$$

Received August 11, 1998.

2000 Mathematics Subject Classification. Primary 34C05, 92D25; Secondary 34C07, 34C23.

Key words and phrases. Predator-prey system, limit cycle, Holling-type functional response, Liénard system.

E-mail address: jsugie@riko.shimane-u.ac.jp 
and

$$
k>\lambda_{p}
$$

system (1.1) has the only equilibrium $\left(\lambda_{p}, \nu_{p}\right)$ in the region $\{(x, y): x>0$ and $y>0\}$. If assumptions (1.2) and (1.3) fail, then system (1.1) has no positive equilibria and, therefore, no limit cycles of (1.1) exist. Hence, it is reasonable to assume (1.2) and (1.3) in discussions about the existence of a unique limit cycle in system (1.1).

The author, Kohno, and Miyazaki [9] gave the following result on the uniqueness of limit cycles of (1.1).

Theorem A. Let $p$ be a positive number with $0<p \leq 1$ or $p \geq 2$. If (1.2), (1.3), and

$$
(p D-(p-2) \mu) \lambda_{p}<(p D-(p-1) \mu) k
$$

are satisfied, then system (1.1) has a unique limit cycle.

They proved Theorem A by means of the well-known uniqueness theorem in Kuang and Freedman [6]. Also, they pointed out that if

$$
1<p<2
$$

then the theorem in [6] is of no use.

The purpose of this paper is to show that (1.2)-(1.4) are sufficient conditions for the uniqueness of limit cycles of (1.1) even in the case (1.5). The proof of our main result is divided into two parts: the existence of at least one limit cycle; the uniqueness of the limit cycle.

To prove the first part, in Sec. 2, we examine the behavior of trajectories of a Liénardtype system, which is equivalent to system (1.1), and construct a Poincaré-Bendixson domain. Applying a result on the existence of at most one limit cycle by Zhang and Gao [13], we prove the second part in Sec. 3.

In the final section, we mention that under the assumptions (1.2) and (1.3), system (1.1) has exactly one limit cycle if and only if condition (1.4) is satisfied.

2. Liénard plane analysis. In this and the next sections, we will prove the following result concerning the uniqueness of limit cycles of (1.1).

Theorem 2.1. Assume (1.2), (1.3), and (1.5). If (1.4) is satisfied, then system (1.1) has a unique limit cycle.

Changing variables

$$
u=x-\lambda_{p}, \quad v=\log \nu_{p}-\log y, \quad d \tau=\frac{x^{p}}{a+x^{p}} d t,
$$

we can transform system (1.1) into the system of Liénard type

$$
\begin{aligned}
& \frac{d u}{d \tau}=\nu_{p}\left(1-e^{-v}\right)-F_{k}(u), \\
& \frac{d v}{d \tau}=-g(u),
\end{aligned}
$$


where

$$
F_{k}(u)=\nu_{p}-r\left(1-\frac{u+\lambda_{p}}{k}\right) \frac{a+\left(u+\lambda_{p}\right)^{p}}{\left(u+\lambda_{p}\right)^{p-1}}
$$

and

$$
g(u)=\mu-D-\frac{a D}{\left(u+\lambda_{p}\right)^{p}} .
$$

The functions $F_{k}(u)$ and $g(u)$ are defined for $u>-\lambda_{p}$. Using the fact that $\lambda_{p}^{p}=$ $a D /(\mu-D)$ and $\nu_{p}=r \mu\left(1-\lambda_{p} / k\right) \lambda_{p} / D$, we have

$$
F_{k}(0)=\nu_{p}-r\left(1-\frac{\lambda_{p}}{k}\right) \lambda_{p}\left(\frac{a}{\lambda_{p}^{p}}+1\right)=0
$$

and

$$
u g(u)=a D\left(\frac{1}{\lambda_{p}^{p}}-\frac{1}{\left(u+\lambda_{p}\right)^{p}}\right) u>0 \quad \text { if } u \neq 0 .
$$

Hence, the origin is a unique critical point of (2.1). Define

$$
G(u)=\int_{0}^{u} g(s) d s=(\mu-D) u+\frac{a D}{p-1}\left(\frac{1}{\left(u+\lambda_{p}\right)^{p-1}}-\frac{1}{\lambda_{p}^{p-1}}\right) .
$$

Noticing $p>1$, we see that

$$
\begin{array}{rll}
\lim _{u \rightarrow-\lambda_{p}} F_{k}(u)=-\infty & \text { and } & \lim _{u \rightarrow \infty} F_{k}(u)=\infty \\
\lim _{u \rightarrow-\lambda_{p}} G(u)=\infty & \text { and } & \lim _{u \rightarrow \infty} G(u)=\infty
\end{array}
$$

We get

$$
F_{k}^{\prime}(u)=-\frac{r}{k}\left\{\left(1-\frac{a(p-1)}{\left(u+\lambda_{p}\right)^{p}}\right) k-2\left(u+\lambda_{p}\right)-a(2-p)\left(u+\lambda_{p}\right)^{1-p}\right\}
$$

for $u>-\lambda_{p}$. Hence, by assumption (1.4), we have

$$
\begin{aligned}
F_{k}^{\prime}(0) & =-\frac{r}{k}\left\{\left(1-\frac{a(p-1)}{\lambda_{p}^{p}}\right) k-2 \lambda_{p}-a(2-p) \lambda_{p}^{1-p}\right\} \\
& =-\frac{r}{k}\left\{\left(1-\frac{(p-1)(\mu-D)}{D}\right) k-2 \lambda_{p}-\frac{(2-p)(\mu-D)}{D} \lambda_{p}\right\} \\
& =-\frac{r}{k D}\left\{(p D-(p-1) \mu) k-(p D-(p-2) \mu) \lambda_{p}\right\}<0 .
\end{aligned}
$$

We will show that system (2.1) has at least one limit cycle that is stable. For the sake of convenience, we denote by $\gamma^{+}(P)$ and $\gamma^{-}(P)$ the positive semitrajectory and the negative semitrajectory of $(2.1)$, respectively, starting at a point $P$ in the domain

$$
D=\left\{(u, v): u>-\lambda_{p} \text { and } v \in \mathbf{R}\right\} \text {. }
$$

We divide the domain $D$ as follows:

$$
\begin{aligned}
& D^{+}=\left\{(u, v): u \geq k-\lambda_{p} \text { and } v \in \mathbf{R}\right\} \\
& D^{-}=\left\{(u, v):-\lambda_{p}<u<k-\lambda_{p} \text { and } v \in \mathbf{R}\right\} .
\end{aligned}
$$

Consider the curve

$$
v=C(u) \stackrel{\text { def }}{=} \log \nu_{p}-\log \left(\nu_{p}-F_{k}(u)\right)
$$


for $-\lambda_{p}<u<k-\lambda_{p}$. Then, by (2.2), (2.4), and (2.6), we see that

$$
\begin{gathered}
C(0)=0 \quad \text { and } C^{\prime}(0)<0 \\
\lim _{u \rightarrow-\lambda_{p}} C(u)=-\infty \quad \text { and } \quad \lim _{u \rightarrow k-\lambda_{p}} C(u)=\infty .
\end{gathered}
$$

Note that the curve $v=C(u)$ is contained in the domain $D^{-}$. If a trajectory of $(2.1)$ meets the curve $v=C(u)$, then it traverses the curve vertically.

Proof of Theorem 2.1 (Existence of limit cycles). Let $P_{1}$ be a point on the line $u=$ $k-\lambda_{p}$. Since

$$
F_{k}(u)>\nu_{p} \text { for } u \geq k-\lambda_{p},
$$

the negative semitrajectory $\gamma^{-}\left(P_{1}\right)$ keeps on running from left to right and, therefore, it stays in the domain $D^{+}$. On the other hand, taking account of $(2.7),(2.8)$, and the vector field of (2.1), we see that the positive semitrajectory $\gamma^{+}\left(P_{1}\right)$

(i) has no vertical asymptotes,

(ii) does not approach the origin directly as $\tau \rightarrow \infty$,

(iii) crosses the curve $v=C(u)$ infinitely many times.

Hence, $\gamma^{+}\left(P_{1}\right)$ remains in the domain $D^{-}$and keeps on rotating around the origin clockwise. Let $P_{2}$ and $P_{3}$ be the first intersecting points of $\gamma^{+}\left(P_{1}\right)$ with the negative $v$-axis and the positive $v$-axis, respectively, and let $P_{4}$ be the second intersecting point of $\gamma^{+}\left(P_{1}\right)$ with the negative $v$-axis. Because of the uniqueness of solutions of $(2.1)$, the point $P_{4}$ lies above $P_{2}$. We denote by $R_{1}$ the region that is enclosed by the $\operatorname{arc} P_{2} P_{4}$ of $\gamma^{+}\left(P_{1}\right)$ and the line segment $P_{2} P_{4}$.

We next examine the behavior of trajectories of (2.1) near the origin. Define

$$
V(u, v)=U(v)+G(u),
$$

where $U(v)=\nu_{p}\left(e^{-v}+v-1\right)$. Then, by (2.3) and (2.5), we see that $V(u, v)$ is a continuous increasing positive definite function and, therefore, the curve $V(u, v)=U\left(v_{0}\right)$ is an oval surrounding the origin for any $v_{0} \in \mathbf{R}$ and the oval converges to the origin as $v_{0} \rightarrow 0$. Also, by (2.2) and (2.6), the derivative of $V$ along a solution of (2.1) satisfies

$$
\dot{V}_{(2.1)}(u, v)=-g(u) F_{k}(u)>0
$$

for $|u|$ small enough. Let $Q_{1}=\left(0, q_{1}\right)$ with $q_{1}>0$ sufficiently small. Then, by $(2.9)$, the negative semitrajectory $\gamma^{-}\left(Q_{1}\right)$ remains in the bounded region that is enclosed by the oval $V(u, v)=U\left(q_{1}\right)$.

There are two cases to consider. If $\gamma^{-}\left(Q_{1}\right)$ rotates around the origin counterclockwise and returns to the positive $v$-axis at a point $Q_{2}=\left(0, q_{2}\right)$, then

$$
q_{2}<q_{1}
$$

In fact, by (2.9), we have

$$
U\left(q_{2}\right)=V\left(0, q_{2}\right)<V\left(0, q_{1}\right)=U\left(q_{1}\right) .
$$

Assume $\gamma^{-}\left(Q_{1}\right)$ does not rotate around the origin. Then it approaches the origin through only the region $\{(u, v): u<0$ and $0<v<C(u)\}$ as $\tau \rightarrow \infty$, or it passes a point on the negative $v$-axis and then approaches the origin through only the region $\{(u, v): u>0$ and $C(u)<v<0\}$ as $\tau \rightarrow \infty$. By (2.8) and the uniqueness of solutions of (2.1), 
the positive semitrajectory $\gamma^{+}\left(Q_{1}\right)$ goes around the origin clockwise and returns to the positive $v$-axis at a point $Q_{0}$ that lies above $Q_{1}$. In the former case, we denote by $R_{2}$ the region that is enclosed by the arc $Q_{1} Q_{2}$ of $\gamma^{-}\left(Q_{1}\right)$ and the line segment $Q_{1} Q_{2}$. In the latter case, we denote by $R_{2}$ the region that is enclosed by the arc $Q_{1} Q_{0}$ of $\gamma^{+}\left(Q_{1}\right)$ and the line segment $Q_{1} Q_{0}$.

Now, we consider the deformed annulus $R_{1} \backslash R_{2}$. Then, by the argument above, we see that for each point $P \in R_{1} \backslash R_{2}$ the positive semitrajectory $\gamma^{+}(P)$ must remain in $R_{1} \backslash R_{2}$. Hence, the annulus is a Poincaré-Bendixson domain. We therefore conclude that system (2.1) has at least one stable limit cycle by means of the Poincaré-Bendixson theorem.

Recall that system (1.1) is equivalent to system (2.1). Hence, system (1.1) also has at least one limit cycle. Thus, the proof of existence of a limit cycle of (1.1) is now complete.

3. Uniqueness of limit cycles. Consider the generalized Liénard system

$$
\begin{aligned}
& \frac{d u}{d \tau}=\varphi(v)-\Gamma(u), \\
& \frac{d v}{d \tau}=-\delta(u),
\end{aligned}
$$

in which $\Gamma(u)$ and $\delta(u)$ are continuously differentiable on $(\alpha, \omega)$, where $\alpha<0<\omega$, and $\varphi(v)$ is continuously differentiable on $\mathbf{R}$. We make the standard assumptions:

$$
\begin{gathered}
\Gamma(0)=0 \text { and } u \delta(u)>0 \text { for } u \neq 0, \\
\varphi(v) \text { is increasing for } v \in \mathbf{R} \text { and } v \varphi(v)>0 \text { if } v \neq 0 .
\end{gathered}
$$

Let $u_{1}(z)$ (resp., $u_{2}(z)$ ) be the inverse function of $z=\Delta(u) \stackrel{\text { def }}{=} \int_{0}^{u} \delta(s) d s$ for $u>0$ (resp., $u<0$ ), and denote $\Gamma_{1}(z)=\Gamma\left(u_{1}(z)\right)$ and $\Gamma_{2}(z)=\Gamma\left(u_{2}(z)\right)$.

In this section, we will give the remainder of the proof of Theorem 2.1. To this end, we use the following result on the existence of at most one limit cycle of (3.1), which is given by Zhang and Gao [13] (see also [11, Theorem 3]).

Theorem B. Assume (3.2) and (3.3). Suppose that

(i) there exists $b \geq 0$ such that $\Gamma_{1}(z) \leq 0 \leq \Gamma_{2}(z)$ for $0 \leq z \leq b$ and $\Gamma_{1}^{\prime}(z) \geq 0$ for $z \geq b$, and $\Gamma_{1}(z) \not \equiv \Gamma_{2}(z)$ for $0<z \ll 1$,

(ii) $\Gamma_{1}(z) / z$ is nondecreasing for $z>b$,

(iii) for every constant $c \geq 1$, if $\Omega_{c}(z)=\Gamma_{2}(w)$ with $b / c<z<w$, then $\Omega_{c}^{\prime}(z) \geq \Gamma_{2}^{\prime}(w)$, where

$$
\Omega_{c}(z)=\frac{1}{c} \Gamma_{1}(c z),
$$

(iv) $\varphi^{\prime}(v) /|\varphi(v)|$ is nonincreasing as $|v|$ increases.

Then system (3.1) has at most one limit cycle.

By virtue of Theorem B, we can give the following.

Proposition 3.1. Suppose that (1.4) is satisfied for $p>1$. If $F_{k}(u) / G(u)$ is nondecreasing for $u>-\lambda_{p}$ and $u \neq 0$, then system (1.1) has at most one limit cycle. 
Proof. Comparing system (3.1) with system (2.1) which is equivalent to (1.1), we see that $\alpha=-\lambda_{p}, \omega=\infty$,

$$
\varphi(v)=\nu_{p}\left(1-e^{-v}\right), \quad \Gamma(u)=F_{k}(u), \quad \text { and } \quad \delta(u)=g(u) .
$$

Hence, it follows from (2.2) and (2.3) that assumption (3.2) is satisfied. It is clear that assumption (3.3) holds.

Remember that if $p>1$ and (1.4) is satisfied, then $F_{k}(u)$ has the properties (2.4) and (2.6). Because of (2.4) and (2.6), the sets $\left\{u>0: F_{k}(u) \leq 0\right\}$ and $\left\{u<0: F_{k}(u) \geq 0\right\}$ are bounded and nonempty. Let

$$
u^{*}=\sup \left\{u>0: F_{k}(u) \leq 0\right\} \quad \text { and } \quad u_{*}=\inf \left\{u<0: F_{k}(u) \geq 0\right\} .
$$

Then $F_{k}\left(u^{*}\right)=F_{k}\left(u_{*}\right)=0, F_{k}^{\prime}\left(u^{*}\right) \geq 0, F_{k}^{\prime}\left(u_{*}\right) \geq 0$,

$$
F_{k}(u)>0 \text { for } u>u^{*} \text {, and } F_{k}(u)<0 \text { for }-\lambda_{p}<u<u_{*} \text {. }
$$

Suppose that $F_{k}(u) / G(u)$ is nondecreasing for $u>-\lambda_{p}$ and $u \neq 0$, that is,

$$
F_{k}^{\prime}(u) G(u) \geq F_{k}(u) g(u) \text { for } u>-\lambda_{p} \text { and } u \neq 0 .
$$

Then, by (3.4), we get

$$
F_{k}^{\prime}(u)>0 \text { for } u>u^{*} \quad \text { and } \quad F_{k}^{\prime}(u)>0 \text { for }-\lambda_{p}<u<u_{*},
$$

and hence, we see that

$$
u F_{k}(u)<0 \text { for } u_{*}<u<u^{*} \text { and } u \neq 0 .
$$

Thus, we conclude that $F_{k}(u)$ has only three zeros: $u=0, u=u^{*}$, and $u=u_{*}$.

We consider the case that $G\left(u^{*}\right) \leq G\left(u_{*}\right)$. Let $b=G\left(u^{*}\right)$. Then, from (3.4)-(3.6) it turns out that assumption (i) in Theorem B is satisfied. Define

$$
F_{1}(z)=F_{k}\left(u_{1}(z)\right) \text { and } F_{2}(z)=F_{k}\left(u_{2}(z)\right)
$$

where $u_{1}(z)$ and $u_{2}(z)$ are the inverse functions of $z=G(u)$ for $u>0$ and for $u<0$, respectively. Then we have

$$
\frac{F_{1}(z)}{z}=\frac{F_{k}\left(u_{1}(z)\right)}{z}=\frac{F_{k}(u)}{G(u)} \quad \text { for } u>0 .
$$

Since $F_{k}(u) / G(u)$ is nondecreasing and $G(u)$ is increasing for $u>0$, we see that $F_{1}(z) / z$ is also nondecreasing for $z>0$. Hence, assumption (ii) holds, and we obtain

$$
F_{1}^{\prime}(z) \geq \frac{F_{1}(z)}{z} \text { for } z>0
$$

Similarly, since $F_{k}(u) / G(u)$ is nondecreasing for $-\lambda<u<0$, the function $F_{2}(z) / z$ is nonincreasing for $z>0$ and

$$
F_{2}^{\prime}(z) \leq \frac{F_{2}(z)}{z} \quad \text { for } z>0
$$

For every constant $c \geq 1$, let

$$
\Omega_{c}(z)=\frac{1}{c} F_{1}(c z)
$$


From (3.4) we see that

$$
\Omega_{c}(z)>0 \text { for } z>\frac{b}{c} .
$$

The function $\Omega_{c}(z) / z$ is nondecreasing for $z>0$. In fact, by (3.7), we have

$$
\frac{d}{d z}\left(\frac{\Omega_{c}(z)}{z}\right)=\frac{d}{d z}\left(\frac{F_{1}(c z)}{c z}\right)=\frac{c z F_{1}^{\prime}(c z)-F_{1}(c z)}{c z^{2}}>0
$$

for $z>0$. Hence, we obtain

$$
\Omega_{c}^{\prime}(z) \geq \frac{\Omega_{c}(z)}{z} \text { for } z>0 .
$$

If $\Omega_{c}(z)=F_{2}(w)$ with $b / c<z<w$, then we get

$$
\Omega_{c}^{\prime}(z) \geq \frac{\Omega_{c}(z)}{z}=\frac{F_{2}(w)}{z}>\frac{F_{2}(w)}{w} \geq F_{2}^{\prime}(w)
$$

by (3.8)-(3.10). Thus, assumption (iii) is satisfied. Since $\varphi^{\prime}(v)=\nu_{p} e^{-v}$, we have

$$
\frac{\varphi^{\prime}(v)}{|\varphi(v)|}=\frac{e^{-v}}{\left|1-e^{-v}\right|} \quad \text { for } v \in \mathbf{R} \text {. }
$$

Hence, assumption (iv) also holds.

Next, we consider the case that $G\left(u^{*}\right)>G\left(u_{*}\right)$. By the transformation $(\tau, u, v) \mapsto$ $(\tau,-u,-v)$, system $(2.1)$ becomes

$$
\begin{aligned}
& \frac{d u}{d \tau}=\tilde{\varphi}(v)-\widetilde{F}_{k}(u), \\
& \frac{d v}{d \tau}=-\tilde{g}(u),
\end{aligned}
$$

where $\tilde{\varphi}(v)=\nu_{p}\left(e^{v}-1\right), \widetilde{F}_{k}(u)=-F_{k}(-u)$, and $\tilde{g}(u)=-g(-u)$. Compare system (3.1) with system (3.11). Then it turns out that $\alpha=-\infty, \omega=\lambda_{p}$,

$$
\varphi(v)=\tilde{\varphi}(v), \quad \Gamma(u)=\widetilde{F}_{k}(u), \quad \text { and } \quad \delta(u)=\tilde{g}(u) .
$$

Hence, assumptions (3.2) and (3.3) are satisfied. Let $\tilde{u}_{1}(z)$ and $\tilde{u}_{2}(z)$ be the inverse functions of $z=\widetilde{G}(u) \stackrel{\text { def }}{=} \int_{0}^{u} \tilde{g}(s) d s=G(-u)$ for $u>0$ and for $u<0$, respectively, and define

$$
\begin{gathered}
\tilde{u}^{*}=\sup \left\{u>0: \widetilde{F}_{k}(u) \leq 0\right\}, \quad \tilde{u}_{*}=\inf \left\{u<0: \widetilde{F}_{k}(u) \geq 0\right\}, \\
\widetilde{F}_{1}(z)=F\left(\tilde{u}_{1}(z)\right), \quad \text { and } \quad \widetilde{F}_{2}(z)=F\left(\tilde{u}_{2}(z)\right) .
\end{gathered}
$$

Then we see that $\tilde{u}_{1}(z)=u_{2}(z), \tilde{u}_{2}(z)=u_{1}(z), \tilde{u}^{*}=-u_{*}, \tilde{u}_{*}=-u^{*}, \widetilde{F}_{1}(z)=F_{2}(z)$, and $\widetilde{F}_{2}(z)=F_{1}(z)$. Since $F_{k}(u) / G(u)$ is nondecreasing for $u>-\lambda$ and $u \neq 0$, we also see that $\widetilde{F}_{k}(u) / \widetilde{G}(u)$ is nondecreasing for $u<\lambda$ and $u \neq 0$. Consequently, in the same manner as in the case that $G\left(u^{*}\right) \leq G\left(u_{*}\right)$, we can show that assumptions (i)-(iii) in Theorem B are satisfied with $b=G\left(u_{*}\right)$. Since

$$
\frac{\tilde{\varphi}^{\prime}(v)}{|\tilde{\varphi}(v)|}=\frac{e^{v}}{\left|e^{v}-1\right|} \quad \text { for } v \in \mathbf{R},
$$

assumption (iv) is also satisfied. 
Thus, in either case, from Theorem B we conclude that system (1.1) has at most one limit cycle. The proof is now complete.

Recall

$$
F_{k}(u)=\nu_{p}-r\left(1-\frac{u+\lambda_{p}}{k}\right) \frac{a+\left(u+\lambda_{p}\right)^{p}}{\left(u+\lambda_{p}\right)^{p-1}}
$$

and

$$
G(u)=(\mu-D) u+\frac{a D}{p-1}\left(\frac{1}{\left(u+\lambda_{p}\right)^{p-1}}-\frac{1}{\lambda_{p}^{p-1}}\right),
$$

and define

$$
H_{k}(u)=F_{k}^{\prime}(u) G(u)-F_{k}(u) G^{\prime}(u)
$$

for $u>-\lambda_{p}$. Then we have

$$
H_{k}(0)=0
$$

Since

$$
F_{k}^{\prime}(u)=-\frac{r}{k}\left\{\left(1-\frac{a(p-1)}{\left(u+\lambda_{p}\right)^{p}}\right) k-2\left(u+\lambda_{p}\right)-a(2-p)\left(u+\lambda_{p}\right)^{1-p}\right\}
$$

and

$$
G^{\prime}(u)=\mu-D-\frac{a D}{\left(u+\lambda_{p}\right)^{p}},
$$

a straightforward calculation yields

$$
\begin{aligned}
H_{k}(u)= & \frac{r(u-D)}{k}\left(u+\lambda_{p}\right)^{2}-\frac{2 p r(\mu-D) \lambda_{p}}{k(p-1)}\left(u+\lambda_{p}\right) \\
& +\frac{a r}{k(p-1)}\left\{\left(p^{2}-p+2\right) D-(p-1)^{2} \mu\right\}\left(u+\lambda_{p}\right)^{2-p} \\
& +\frac{\mu-D}{p-1}\left\{p r \lambda_{p}-(p-1) \nu_{p}\right\}+\frac{a^{2} r D}{k(p-1)}\left(u+\lambda_{p}\right)^{2-2 p} \\
& -\frac{a p r}{k(p-1)}\left\{(p D-(p-1) \mu) k+(2-p)(\mu-D) \lambda_{p}\right\}\left(u+\lambda_{p}\right)^{1-p} \\
& +\frac{a r \lambda_{p}}{k}\left\{(p D-(p-1) \mu) k-\mu \lambda_{p}\right\}\left(u+\lambda_{p}\right)^{-p} .
\end{aligned}
$$

Lemma 3.1. Assume (1.4) and (1.5). Then we have the relations

$$
p D>(p-1) \mu
$$

and

$$
\left(p^{2}-p+2\right) D>(p-1)^{2} \mu .
$$

Proof. From (1.5) we see that

$$
p D-(p-2) \mu>0 .
$$

Hence, together with (1.4), we get (3.14). Using (3.14), we have

$$
\begin{aligned}
\left(p^{2}-p+2\right) D-(p-1)^{2} \mu & >\left(p^{2}-p+2\right) D-(p-1) p D \\
& =2 D>0 .
\end{aligned}
$$


The lemma is proved.

Differentiate (3.13) to obtain

$$
\begin{aligned}
H_{k}^{\prime}(u)= & \frac{2 r(\mu-D)}{k}\left(u+\lambda_{p}\right)-\frac{2 p r(\mu-D) \lambda_{p}}{k(p-1)} \\
& +\frac{a(2-p) r}{k(p-1)}\left\{\left(p^{2}-p+2\right) D-(p-1)^{2} \mu\right\}\left(u+\lambda_{p}\right)^{1-p} \\
& +\frac{a p r}{k}\left\{(p D-(p-1) \mu) k+(2-p)(\mu-D) \lambda_{p}\right\}\left(u+\lambda_{p}\right)^{-p} \\
& -\frac{2 a^{2} r D}{k}\left(u+\lambda_{p}\right)^{1-2 p}-\frac{a p r \lambda_{p}}{k}\left\{(p D-(p-1) \mu) k-\mu \lambda_{p}\right\}\left(u+\lambda_{p}\right)^{-1-p} .
\end{aligned}
$$

Then, from the fact that $\lambda_{p}^{p}=a D /(\mu-D)$ we have

$$
\begin{aligned}
H_{k}^{\prime}(0)= & \frac{r(\mu-D)}{k}\left\{2 \lambda_{p}-\frac{2 p}{p-1} \lambda_{p}+\frac{2-p}{(p-1) D}\left\{\left(p^{2}-p+2\right) D-(p-1)^{2} \mu\right\} \lambda_{p}\right. \\
+ & \frac{p}{D}\left\{(p D-(p-1) \mu) k+(2-p)(\mu-D) \lambda_{p}\right\} \\
& \left.-\frac{2(\mu-D)}{D} \lambda_{p}-\frac{p}{D}\left\{(p D-(p-1) \mu) k-\mu \lambda_{p}\right\}\right\}
\end{aligned}
$$

Now, we define

$$
k^{*}=\frac{p D-(p-2) \mu}{p D-(p-1) \mu} \lambda_{p}
$$

It then follows from (3.14) that condition (1.4) is equivalent to

$$
k^{*}<k \text {. }
$$

LEMma 3.2. The function $H_{k^{*}}^{\prime}(u)$ is increasing for $u>-\lambda_{p}$ and satisfies

$$
u H_{k^{*}}^{\prime}(u)>0 \text { if } u \neq 0 .
$$

Proof. Since $H_{k^{*}}^{\prime}(0)=0$, it suffices to show that $H_{k^{*}}^{\prime \prime}(u)>0$ for $u>-\lambda_{p}$ and $u \neq 0$. We get

$$
\begin{aligned}
H_{k^{*}}^{\prime \prime}(u)= & \frac{2 r(\mu-D)}{k^{*}}-\frac{a(2-p) r}{k^{*}}\left\{\left(p^{2}-p+2\right) D-(p-1)^{2} \mu\right\}\left(u+\lambda_{p}\right)^{-p} \\
& -\frac{a p^{2} r}{k^{*}}\left\{(p D-(p-1) \mu) k^{*}+(2-p)(\mu-D) \lambda_{p}\right\}\left(u+\lambda_{p}\right)^{-1-p} \\
& +\frac{2 a^{2}(2 p-1) r D}{k^{*}}\left(u+\lambda_{p}\right)^{-2 p} \\
& +\frac{a p(p+1) r \lambda_{p}}{k^{*}}\left\{(p D-(p-1) \mu) k^{*}-\mu \lambda_{p}\right\}\left(u+\lambda_{p}\right)^{-2-p}
\end{aligned}
$$


for $u>-\lambda_{p}$. Hence, we have

$$
\begin{aligned}
& H_{k^{*}}^{\prime \prime}(0)=\frac{r(\mu-D)}{k^{*} D}\left\{2 D-(2-p)\left\{\left(p^{2}-p+2\right) D-(p-1)^{2} \mu\right\}\right. \\
& -\frac{p^{2}}{\lambda_{p}}\left\{(p D-(p-1) \mu) k^{*}+(2-p)(\mu-D) \lambda_{p}\right\} \\
& \left.+2(2 p-1)(\mu-D)+\frac{p(p+1)}{\lambda_{p}}\left\{(p D-(p-1) \mu) k^{*}-\mu \lambda_{p}\right\}\right\} \\
& =\frac{r(\mu-D)}{k^{*} D \lambda_{p}}\left\{\lambda _ { p } \left(2 D+(2-p)(p-1)^{2} \mu-(2-p)\left(p^{2}-p+2\right) D\right.\right. \\
& \left.-p^{2}(2-p)(\mu-D)+2(2 p-1)(\mu-D)-p(p+1) \mu\right) \\
& \left.+k^{*}(p D-(p-1) \mu)\left(-p^{2}+p(p+1)\right)\right\} \\
& =\frac{p r(\mu-D)}{k^{*} D \lambda_{p}}\left\{-(p D-(p-2) \mu) \lambda_{p}+(p D-(p-1) \mu) k^{*}\right\}=0 \text {. }
\end{aligned}
$$

Also we have

$$
H_{k^{*}}^{\prime \prime \prime}(u)=\frac{a p r}{k^{*}\left(u+\lambda_{p}\right)^{2 p+1}} W(u)
$$

where

$$
\begin{aligned}
W(u)= & (2-p)\left\{\left(p^{2}-p+2\right) D-(p-1)^{2} \mu\right\}\left(u+\lambda_{p}\right)^{p} \\
& +p(p+1)\left\{(p D-(p-1) \mu) k^{*}+(2-p)(\mu-D) \lambda_{p}\right\}\left(u+\lambda_{p}\right)^{p-1} \\
& -4 a(2 p-1) D-(p+1)(p+2) \lambda_{p}\left\{(p D-(p-1) \mu) k^{*}-\mu \lambda_{p}\right\}\left(u+\lambda_{p}\right)^{p-2}
\end{aligned}
$$

for $u>-\lambda_{p}$. From (3.14) we see that

$$
\begin{aligned}
(p D-(p-1) \mu) k^{*} & =(p D-(p-2) \mu) \lambda_{p}-\mu \lambda_{p} \\
& =(p D-(p-1) \mu) \lambda_{p}>0 .
\end{aligned}
$$

Hence, together with (1.2), (1.5), (3.14), and (3.15), we obtain

$$
\begin{aligned}
W^{\prime}(u)= & p(2-p)\left\{\left(p^{2}-p+2\right) D-(p-1)^{2} \mu\right\}\left(u+\lambda_{p}\right)^{p-1} \\
& +p(p+1)(p-1)\left\{(p D-(p-1) \mu) k^{*}+(2-p)(\mu-D) \lambda_{p}\right\}\left(u+\lambda_{p}\right)^{p-2} \\
& +(p+1)(p+2)(2-p) \lambda_{p}\left\{(p D-(p-1) \mu) k^{*}-\mu \lambda_{p}\right\}\left(u+\lambda_{p}\right)^{p-3} \\
> & 0
\end{aligned}
$$

for $u>-\lambda_{p}$. Hence, noticing

$$
W(0)=\frac{2 a(p+1) D}{(\mu-D) \lambda_{p}}\left\{(p D-(p-2) \mu) \lambda_{p}-(p D-(p-1) \mu) k^{*}\right\}=0,
$$

we conclude that

$$
W(u)<0 \text { for }-\lambda_{p}<u<0 \text { and } W(u)>0 \text { for } u>0 .
$$

Note that the signs of $W(u)$ and $H_{k^{*}}^{\prime \prime \prime}(u)$ are the same. It then follows from (3.18) that $H_{k^{*}}^{\prime \prime}(u)>0$ for $u>-\lambda_{p}$ and $u \neq 0$. 
Thus, the proof of Lemma 3.2 is complete.

We are now ready to give the remainder of the proof of Theorem 2.1 .

Proof of Theorem 2.1 (Uniqueness of limit cycles). As shown in Sec. 2, system (1.1) has at least one limit cycle. Hence, by Proposition 3.1, we can prove Theorem 2.1 if only we show that $F_{k}(u) / G(u)$ is nondecreasing for $u>-\lambda_{p}$ and $u \neq 0$. Since

$$
\frac{d}{d u}\left(\frac{F_{k}(u)}{G(u)}\right)=\frac{H_{k}(u)}{\{G(u)\}^{2}},
$$

it is sufficient to show that $H_{k}(u)$ is positive for $u>-\lambda_{p}$ and $u \neq 0$.

We can rewrite $H_{k}^{\prime}(u)$ as

$$
H_{k}^{\prime}(u)=\frac{r}{k} K(u)+L(u)
$$

where

$$
\begin{aligned}
K(u)= & 2(\mu-D)\left(u+\lambda_{p}\right)-\frac{2 p(\mu-D)}{p-1} \lambda_{p} \\
& +\frac{a(2-p)}{p-1}\left\{\left(p^{2}-p+2\right) D-(p-1)^{2} \mu\right\}\left(u+\lambda_{p}\right)^{1-p} \\
& +a p(2-p)(\mu-D) \lambda_{p}\left(u+\lambda_{p}\right)^{-p} \\
& -2 a^{2} D\left(u+\lambda_{p}\right)^{1-2 p}+a p \mu \lambda_{p}^{2}\left(u+\lambda_{p}\right)^{-1-p}
\end{aligned}
$$

and

$$
L(u)=\frac{\operatorname{apr}(p D-(p-1) \mu)}{\left(u+\lambda_{p}\right)^{p+1}} u .
$$

It is clear that

$$
\begin{aligned}
K^{\prime}(u)= & 2(\mu-D)-a(2-p)\left\{\left(p^{2}-p+2\right) D-(p-1)^{2} \mu\right\}\left(u+\lambda_{p}\right)^{-p} \\
& -a p^{2}(2-p)(\mu-D) \lambda_{p}\left(u+\lambda_{p}\right)^{-1-p}+2 a^{2}(2 p-1) D\left(u+\lambda_{p}\right)^{-2 p} \\
& -a p(p+1) \mu \lambda_{p}^{2}\left(u+\lambda_{p}\right)^{-2-p}
\end{aligned}
$$

and

$$
u L(u)>0
$$

for $u>-\lambda_{p}$ and $u \neq 0$. Define

$$
I=\left\{u>-\lambda_{p}: K(u) \geq 0\right\} \quad \text { and } \quad J=\left\{u>-\lambda_{p}: K(u)<0\right\} .
$$

Using (1.2), (1.3), (1.5), (3.16), (3.19), and (3.20), we have

$$
K(0)=\frac{k}{r}\left(H_{k}^{\prime}(0)-L(0)\right)=0
$$


and

$$
\begin{aligned}
K^{\prime}(0)= & \frac{(\mu-D)}{D}\left\{2 D-(2-p)\left\{\left(p^{2}-p+2\right) D-(p-1)^{2} \mu\right\}\right. \\
& \left.\quad-p^{2}(2-p)(\mu-D)+2(2 p-1)(\mu-D)-p(p+1) \mu\right\} \\
= & \frac{(\mu-D)}{D}\left\{D\left(2-(2-p)\left(p^{2}-p+2\right)+p^{2}(2-p)-2(2 p-1)\right)\right. \\
& \left.\quad+\mu\left((2-p)(p-1)^{2}-p^{2}(2-p)+2(2 p-1)-p(p+1)\right)\right\} \\
= & -\frac{p(\mu-D)}{D}(p D-(p-2) \mu)<0 .
\end{aligned}
$$

Hence, the intervals $I$ and $J$ are nonempty. Since

$$
\frac{\partial}{\partial k} H_{k}^{\prime}(u)=-\frac{r}{k^{2}} K(u)
$$

we see that if $u \in I$, then $H_{k}^{\prime}(u)$ is nonincreasing with respect to $k$; and if $u \in J$, then $H_{k}^{\prime}(u)$ is increasing with respect to $k$. We divide our argument into two cases.

Case (i) $u \in I$. If $-\lambda_{p}<u<0$, then by (3.17) and Lemma 3.2, we have

$$
H_{k}^{\prime}(u) \leq H_{k^{*}}^{\prime}(u)<0 \text {. }
$$

If $u>0$, then

$$
H_{k}^{\prime}(u) \searrow L(u) \quad \text { as } k \rightarrow \infty
$$

and, therefore,

$$
H_{k}^{\prime}(u) \geq L(u)>0 .
$$

Case (ii) $u \in J$. If $-\lambda_{p}<u<0$, then

$$
H_{k}^{\prime}(u) \nearrow L(u) \text { as } k \rightarrow \infty
$$

and, therefore,

$$
H_{k}^{\prime}(u) \leq L(u)<0 .
$$

If $u>0$, then by (3.17) and Lemma 3.2 again, we have

$$
H_{k}^{\prime}(u)>H_{k^{*}}^{\prime}(u)>0 .
$$

Thus, in either case, we obtain

$$
u H_{k}^{\prime}(u)>0 \text { for } u>-\lambda_{p} \text { and } u \neq 0 .
$$

Hence, from (3.12) we conclude that

$$
H_{k}(u)>0 \text { for } u>-\lambda_{p} \text { and } u \neq 0 .
$$

This completes the proof.

REMARK 3.1. By a simple computation, we have

$$
K^{\prime \prime}(u)<0 \text { for }-\lambda_{p}<u \leq 0 .
$$

Since $K(0)=0$ and $K^{\prime}(0)<0$, we see that

$$
K(u)>0 \text { for }-\lambda_{p}<u<0 .
$$

Hence, $u \in J$ implies $u \leq 0$. This means that the former of Case (ii) does not occur. 
4. Discussion. Consider again the generalized Liénard system (3.1) under the assumptions (3.2) and (3.3). Then we can state the following modification of Zhang's uniqueness theorem in [12].

Theorem C. Assume (3.2) and (3.3). If

$$
\Gamma^{\prime}(0) \frac{d}{d u}\left(\frac{\Gamma^{\prime}(u)}{\delta(u)}\right)<0 \quad \text { for } u \in(\alpha, \omega) \text { and } u \neq 0,
$$

then system (3.1) has at most one limit cycle.

Theorem $\mathrm{C}$ or similar results are used in many papers to show the uniqueness of limit cycles of predator-prey models. For example, see $[1,4,5,6,7]$. However, we cannot apply Theorem $\mathrm{C}$ to our problem whether system (1.1) with

$$
1<p<2
$$

has exactly one limit cycle. We will show that assumption (4.1) is not satisfied.

As observed previously, the functions $\Gamma(u)$ and $\delta(u)$ correspond to $F_{k}(u)$ and $g(u)$ in system (2.1), respectively. Since

$$
F_{k}(u)=\nu_{p}-r\left(1-\frac{u+\lambda_{p}}{k}\right) \frac{a+\left(u+\lambda_{p}\right)^{p}}{\left(u+\lambda_{p}\right)^{p-1}}
$$

and

$$
g(u)=\mu-D-\frac{a D}{\left(u+\lambda_{p}\right)^{p}}
$$

for $u>-\lambda_{p}$, we get

$$
\begin{aligned}
& F_{k}^{\prime}(u)=-\frac{r}{k}\left\{\left(1-\frac{a(p-1)}{\left(u+\lambda_{p}\right)^{p}}\right) k-2\left(u+\lambda_{p}\right)-a(2-p)\left(u+\lambda_{p}\right)^{1-p}\right\}, \\
& F_{k}^{\prime \prime}(u)=-\frac{r}{k}\left\{\frac{a k p(p-1)}{\left(u+\lambda_{p}\right)^{p+1}}-2+\frac{a(p-1)(2-p)}{\left(u+\lambda_{p}\right)^{p}}\right\},
\end{aligned}
$$

and

$$
g^{\prime}(u)=\frac{a p D}{\left(u+\lambda_{p}\right)^{p+1}} .
$$

By a direct computation, we have

$$
\begin{aligned}
\frac{d}{d u}\left(\frac{\Gamma^{\prime}(u)}{\delta(u)}\right) & =\frac{d}{d u}\left(\frac{F_{k}^{\prime}(u)}{g(u)}\right)=\frac{F_{k}^{\prime \prime}(u) g(u)-F_{k}^{\prime}(u) g^{\prime}(u)}{\{g(u)\}^{2}} \\
& =\frac{a r w(u)}{k\left(u+\lambda_{p}\right)^{2 p}},
\end{aligned}
$$

where

$$
\begin{aligned}
w(u)= & \frac{2 D}{\lambda_{p}^{p}}\left(u+\lambda_{p}\right)^{2 p}-\left\{\left(p^{2}-p+4\right) D+(p-1)(2-p) \mu\right\}\left(u+\lambda_{p}\right)^{p} \\
& +k p(p D-(p-1) \mu)\left(u+\lambda_{p}\right)^{p-1}-a(2-p) D .
\end{aligned}
$$

It is clear that

$$
\lim _{u \rightarrow \infty} w(u)=\infty
$$


On the other hand, from (4.2) we see that

$$
\lim _{u \rightarrow-\lambda_{p}} w(u)=-a(2-p) D<0 .
$$

Thus, $w(u)$ does not have the definite sign and, therefore, assumption (4.1) does not hold.

Let us turn to our problem. Combining Theorem 2.1 with Theorem A, we have the following result.

Theorem 4.1. Assume (1.2) and (1.3). If (1.4) is satisfied, then system (1.1) has a unique limit cycle.

In a recent paper [8], the author and Katayama proved that if

$$
(p D-(p-2) \mu) \lambda_{p} \geq(p D-(p-1) \mu) k,
$$

namely, the negation of (1.4), then system (1.1) has no limit cycles. We therefore conclude that condition (1.4) is necessary and sufficient for the uniqueness of limit cycles of (1.1) under the assumptions (1.2) and (1.3). In other words, the parameters satisfying

$$
(p D-(p-2) \mu) \lambda_{p}=(p D-(p-1) \mu) k
$$

are bifurcation values. As the parameters satisfying (1.4) approach the bifurcation values, the limit cycle of (1.1) becomes smaller and tends to the only equilibrium $\left(\lambda_{p}, \nu_{p}\right)$.

Acknowledgment. This work was partially supported by Grant-in-Aid for Scientific Research 10874028.

\section{REFERENCES}

[1] Jun-Ping Chen and Hong-De Zhang, The qualitative analysis of two species predator-prey model with Holling's type III functional response, Appl. Math. Mech. 7, 77-86 (1986)

[2] Kuo-Shung Cheng, Uniqueness of a limit cycle for a predator-prey system, SIAM J. Math. Anal. 12, 541-548 (1981)

[3] Sun-Hong Ding, On a kind of predator-prey system, SIAM J. Math. Anal. 20, 1426-1435 (1989)

[4] Xun-Cheng Huang, Uniqueness of limit cycles of generalised Liénard systems and predator-prey systems, J. Phys. A: Math. Gen. 21, L685-L691 (1988)

[5] R. E. Kooij and A. Zegeling, Qualitative properties of two-dimensional predator-prey systems, Nonlinear Anal. 29, 693-715 (1997)

[6] Yang Kuang and H. I. Freedman, Uniqueness of limit cycles in Gause-type models of predator-prey system, Math. Biosci. 88, 67--84 (1988)

[7] H. N. Moreira, On Liénard's equation and the uniqueness of limit cycles in predator-prey systems, J. Math. Biol. 28, 341-354 (1990)

[8] J. Sugie and M. Katayama, Global asymptotic stability of a predator-prey system of Holling type, Nonlinear Anal. 38, 105-121 (1999)

[9] J. Sugie, R. Kohno, and R. Miyazaki, On a predator-prey system of Holling type, Proc. Amer. Math. Soc. 125, 2041-2050 (1997)

[10] J. Sugie, K. Miyamoto, and K. Morino, Absence of limit cycles of a predator-prey system with a sigmoid functional response, Appl. Math. Lett. 9, 85-90 (1996)

[11] Xian-Wu Zeng, Zhi-Fen Zhang, and Su-Zhi Gao, On the uniqueness of the limit cycle of the generalized Liénard equation, Bull. London Math. Soc. 26, 213-247 (1994)

[12] Zhi-Fen Zhang, Proof of the uniqueness theorem of limit cycles of generalized Liénard equation, Appl. Anal. 23, 63-74 (1986)

[13] Zhi-Fen Zhang and Su-Zhi Gao, On the uniqueness of the limit cycle of Liénard equation, Acta Math. Peking Univ. 22, 1-13 (1986) 